\title{
Studying SARS-CoV-2 infectivity and therapeutic responses with complex organoids
}

\author{
Kevin G. Chen ${ }^{1 凶}{ }^{\boxplus}$, Kyeyoon Park ${ }^{1}$ and Jason R. Spence ${ }^{2,3,4}$
}

\begin{abstract}
Clinical management of patients with severe complications of COVID-19 has been hindered by a lack of effective drugs and a failure to capture the extensive heterogeneity of the disease with conventional methods. Here we review the emerging roles of complex organoids in the study of SARS-CoV-2 infection, modelling of COVID-19 disease pathology and in drug, antibody and vaccine development. We discuss opportunities for COVID-19 research and remaining challenges in the application of organoids.
\end{abstract}

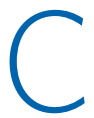
OVID-19, caused by infection with SARS-CoV-2, represents a global health emergency. As of 30 May 2021, approximately 169 million individuals have been infected and 3,530,582 deaths from COVID-19 have been confirmed worldwide ${ }^{1}$. Even though vaccines have now been established to prevent infection, to date, no specific antiviral drugs exist that target SARS-CoV-2 to mitigate established disease. Clinical management of patients with COVID-19 focuses mainly on improving symptoms, supporting lung function, preventing a sudden acute increase of circulating cytokines (cytokine storm) and controlling infections ${ }^{2}$.

Ongoing fast-track clinical trials focus on therapeutic solutions that block the SARS-CoV-2 infection cycle and associated pathophysiological processes ${ }^{3}$. Nevertheless, it remains poorly understood how the genetic background of patients with COVID-19 might affect the severity of symptoms $s^{4,5}$. Similarly, whether SARS-CoV-2-host-receptor interactions might differ depending on the age, gender and ethnicity of a patient has not been clarified. As a result, the design of effective vaccines and antiviral drugs has remained challenging. The advancement of organoid-based assays derived from human pluripotent stem cells (hPSCs) and adult stem cells (ASCs) offers an opportunity to expand and bank various types of tissue-specific organoids for biomedical research ${ }^{6-9}$. Accordingly, stem cell-based two-dimensional (2D) cell cultures and 3D organoids are also used to study SARS-CoV- 2 infection ${ }^{10-19}$. These studies highlight the need to define the roles of stem cell-based organoids in COVID-19 research.

In this Review, we recapitulate the SARS-CoV-2 infection cycle and associated intervention strategies. We evaluate current COVID19-based assays, focusing on their strengths and potential limitations. We further elucidate the role of respiratory cell types and lung organoids in assessing SARS-CoV-2 susceptibility and discuss other organoid systems (derived from hPSCs and ASCs) that can be used. Finally, we examine the benefits of organoids in studying SARS-CoV2 -induced pathophysiology and predicting therapeutic outcomes.

\section{SARS-CoV-2 infection cycle and associated intervention strategies}

SARS-CoV-2 is a positive-sense, single-stranded ribonucleic acid (RNA) Betacoronavirus, potentially evolved from a bat coronavirus ${ }^{20-23}$.
Genomic diversity of SARS-CoV-2 in patients with COVID-19 is evident $^{24-26}$, but the environmental SARS-CoV-2 genome is relatively stable $^{27}$. The structural genomics of SARS-CoV-2 indicates evolutionarily conserved functional regions of viral protein ${ }^{27-29}$. In addition, SARS-CoV-2 shares a similar infection cycle with SARS-CoV and Middle East respiratory syndrome coronavirus (MERS-CoV) ${ }^{30-32}$.

SARS-CoV-2 infection cycle. Similar to SARS-CoV, SARS-CoV-2 enters and infects a human host cell via multiple coordinated processes $^{30-32}$. The SARS-CoV-2 infection cycle is shown in Fig. 1a, with distinct steps (1-17), starting from host cell entry via membrane fusion and endocytosis to the release of a mature SARS-CoV-2. In patients with COVID-19, the infection cycle increases the viral load in the respiratory tissues, kidneys and intestine ${ }^{33}$. The induction and release of cellular cytokines (also called a cytokine storm) may trigger a wide range of host immunological and inflammatory responses in these tissues ${ }^{34,35}$ (Fig. 1b-e). Cytokine storms often lead to diffuse alveolar damage, acute respiratory distress syndrome, loss of gas exchange, respiratory failure and multi-organ damage, increasing overall death rates ${ }^{35-40}$.

Therapeutic strategies. Despite an incomplete understanding of the SARS-CoV-2-specific infection cycle, known viral processes could be probed for potential pharmacological, immunological and molecular interventions. Such experimental and clinical interventions have been reported for SARS-CoV-2 $2^{14,41-48}$, some of which are listed in Fig. 1.

The abrogation of viral cell entry effectively prevents viral infection. Blockage of spike glycoprotein (S-gp) binding to the human receptor, angiotensin-converting enzyme 2 (ACE2), using human recombinant soluble ACE2 (hrsACE2) reduced SARS-CoV-2 recovery from Vero cells, resulting in a 1,000- to 5,000-fold reduction in viral growth ${ }^{14}$. This blockage by soluble ACE2 appears to be species-specific, as recombinant mouse ACE2 had no effect ${ }^{14}$. Transmembrane serine protease 2 (TMPRSS2)-mediated S-gp priming can be blocked with camostat, a clinically proven protease inhibitor, and substantially (approximately 88\%) inhibited by an anti-ACE2 antibody ${ }^{41}$ (at $20 \mu \mathrm{g} \mathrm{ml}^{-1}$ ). Sera from patients who had recovered from SARS partially (approximately 45\%) neutralized

${ }^{1} \mathrm{NIH}$ Stem Cell Unit, National Institute of Neurological Disorders and Stroke, National Institutes of Health, Bethesda, MD, USA. ${ }^{2}$ Department of Internal Medicine, University of Michigan Medical School, Ann Arbor, MI, USA. ${ }^{3}$ Department of Cell and Developmental Biology, University of Michigan Medical School, Ann Arbor, MI, USA. ${ }^{4}$ Department of Biomedical Engineering, University of Michigan Medical School, Ann Arbor, MI, USA.

凶e-mail: cheng@mail.nih.gov 
pseudotyped SARS-CoV-2 entry ${ }^{41}$. CR3022, a neutralizing antibody isolated from a patient who had recovered from SARS, targets S-gp receptor-binding domain (S-RBD) of SARS-CoV ${ }^{49}$ and also binds to the SARS-CoV-2 S-RBD ${ }^{50}$. High-resolution structures revealed a mechanism by which neutralizing antibodies, such as CR3022, recognize S-RBD in its trimeric configuration ${ }^{51}$. These studies provide a molecular basis for future therapeutic interventions to prevent SARS-CoV-2 cell entry.

Beyond S-gp-ACE2-mediated membrane fusion, little is known about other potential cell-entry mechanisms of SARS-CoV-2, such as endocytic pathways which are evident in other coronaviruses. These pathways depend on clathrin-dependent endocytosis for SARS-CoV ${ }^{52}$, membrane rafts and caveolar endocytosis for the human coronavirus $229 \mathrm{E}^{53}$, and clathrin- and caveolar-independent entry of feline coronavirus ${ }^{54}$. Successful abolishment of SARS-CoV-2 entry by camostat suggests that the endocytic pathway may not be a major mechanism for SARS-CoV-2 cell entry. However, this finding has to be confirmed in different cellular and animal models.

The United States Food and Drug Administration (FDA)approved drug ivermectin inhibits the replication of SARS-CoV-2 in an in vitro model (Vero-hSLAM cells) ${ }^{46}$. The promising antiviral drug remdesivir (GS-5734), an adenosine analogue, also inhibits SARS-CoV-2 replication ${ }^{44}$. Remdesivir has become the first anti-SARS-CoV-2 drug approved by the FDA after a phase III clinical trial ${ }^{55}$. However, the World Health Organization's solidarity trial revealed that remdesivir neither reduced mortality nor shortened the recovery time of COVID-1948. A less toxic derivative of chloroquine, hydroxychloroquine, is an endosomal acidification inhibitor and is effective in inhibiting SARS-CoV-2 infection in cell culture ${ }^{42}$. Hydroxychloroquine has gained widespread use in the treatment of COVID-19. However, its broader clinical application has been under scrutiny due to the absence of well-controlled data on its effectiveness and reported severe side effects ${ }^{43}$.

Importantly, about $20 \%$ of severe COVID-19 cases are associated with cytokine storms - which also occur in SARS and MERS ${ }^{35,56}$ which can be treated by inhibiting cytokine release or accelerating cytokine clearance in targeted cells ${ }^{57}$. The monoclonal antibody tocilizumab, which inhibits interleukin-6 (IL-6), has been used to treat cytokine storms in patients with COVID-19 in clinical trials ${ }^{58}$. In an early trial, treatment with tocilizumab reduced the risk of invasive mechanical ventilation or death rate in patients with severe COVID-1959. In a later report, patients with moderate COVID-19 treated with tocilizumab showed fewer severe infections than those who received a placebo. However, tocilizumab did not prevent the need for intubation or death in these patients ${ }^{60}$. Thus, the role of tocilizumab in the treatment of COVID-19 remains obscure.
Encouragingly, a meta-analysis of 7 randomized clinical trials revealed lower 28 day mortality among critically ill patients who received systemic corticosteroids compared with those who received usual care or placebos ${ }^{61}$. In the RECOVERY trial, the immunosuppressant dexamethasone (6 $\mathrm{mg}$ once daily for up to 10 days) reduced 28 day mortality in patients who required oxygen, particularly in those receiving mechanical ventilation ${ }^{47}$. No benefit was found for patients who did not require oxygen supplementation ${ }^{47}$. The mechanism that underlies the beneficial effect of dexamethasone in these patients is not well understood. It may be associated with the inhibition of major pro-inflammatory pathways such as NF- $\kappa$ B in the most severe patients ${ }^{38}$ (Fig. 1a,f). Nonetheless, these clinical trials suggest that cytokine storms contribute to lung injury and multi-organ failure in patients with severe COVID-19. For this reason, major health organizations recommend dexamethasone (or potentially other glucocorticoids) as standard care for patients with severe COVID-19.

Other factors that influence the infection cycle. SARS-CoV-2 infection cycles are associated with diverse clinical characteristics in patients with COVID-19 manifesting no symptoms, or mild or severe symptoms such as acute respiratory disease and pneumonia $^{62-64}$. Some asymptomatic patients have persistent negative computed tomographic findings ${ }^{65}$, suggesting low viral load or low inflammatory and immunological responses in the lungs. Approximately $80 \%$ of the infections are asymptomatic or mild, $15 \%$ are severe (requiring oxygen inhaler), and $5 \%$ of patients are in critical condition and require a ventilator ${ }^{66}$. At this time, it is impossible to predict which patient will be in the $5 \%$ that need critical care.

Many tangible intrinsic (such as age, gender and ethnicity) and extrinsic (such as lifestyle) factors influence the infection cycle, morbidity and mortality rates. SARS-CoV-2 infects people of all ages, from neonates to older adults ${ }^{67-69}$. However, paediatric cases are less frequently symptomatic than older adults ${ }^{69-71}$. SARS-CoV-2 infection also affects women less than it affects men $^{72}$, possibly because androgen signalling modulates ACE2 levels. Increased androgen levels are associated with a higher risk of SARS-CoV-2 infection and disease severity in men $^{17}$. Demographic data reveal high morbidity and mortality rates in African Americans in the USA ${ }^{73-75}$, although underlying reasons remain unclear and could likely be multifactorial, including socioeconomic factors and access to healthcare.

Cigarette smoking increases susceptibility to SARS-CoV-2 infections by upregulating ACE2 expression ${ }^{16,76,77}$. Collectively, age, gender, lifestyle and demographic differences might modulate viral receptor expression and other unknown determinants, which, in turn, contribute to disease severity and therapeutic response. For instance, co-expression of ACE2 and TMPRSS2 mRNAs is tightly

Fig. 1 | SARS-CoV-2 infection cycle, immunological response, molecular targets and intervention strategies. a, The infection cycle includes S-gp binding to the human ACE2 receptor, pre-cleavage by the host cellular protease furin to dissociate the S1 subunit from the S2 subunit of S-gp ${ }^{161,162}$, and S2 activation mediated by serine protease TMPRSS2 co-receptor ${ }^{41}$. Notably, cleavage by furin is required for the entry of SARS-CoV-2 into human lung cells ${ }^{161}$. S2 activation triggers viral fusion with the host cell membrane. In the host cell cytoplasm, the positive-sense SARS-CoV-2 genomic RNA is transcribed to yield full-length negative-sense RNAs for genome replication and negative-sense (-) subgenomic RNAs (sgRNAs) for producing subgenomic mRNAs ( $s g$ mRNAs). Subgenomic mRNAs, converted from -sgRNAs, are translated into viral structural proteins, including S-gp, envelope (E), membrane (M), and nucleocapsid $(\mathrm{N})$ proteins $^{30-32}$. Finally, viral genome encapsulation and reassembly enable virus maturation and export out of cells for the next infection cycle. $\mathbf{b}, \mathbf{c}$, SARS-CoV-2 induces immunological responses through viral antigen presentation in macrophages (b), naive T cell activation and release of cytokines $(\mathbf{c})$. d, Possible dual roles of B cell-mediated humoral immune response: $B$ cells generate the neutralizing antibodies to protect the lung from SARS-CoV-2 infection and contribute to cytokine-induced damage through FcyR-mediated and antibody-dependent enhancement of SARS-CoV-2 infection. e, SARS-CoV-2-induced organ damage via an unbalanced presence of pro-inflammatory cytokines or absence of antiviral factors. f, Representative intervention strategies, such as the development of drugs, vaccines, antibodies, recombinant proteins and repurposing of approved drugs against SARS-CoV-2 infection, with molecular targets indicated by numbers in a. ADE, antibody-dependent enhancement; APC, antigen-presenting cells; CXCL10, C-X-C motif chemokine ligand 10; ER, endoplasmic reticulum; FcyR, Fc-gamma receptor; IL-6R, IL-6 receptor; JAK, janus kinase; JAKi, janus kinase inhibitor; NA, data not available; NF- $\mathrm{kB}$, nuclear factor kappa B; Rc, replicase and transcriptase complex; NSPs, non-structural proteins; rc-ACE2-Ig, recombinant ACE2-Ig; STAT, signal transducer and activator of transcription; TMPRSS2, transmembrane protease serine 2; TNF, tumour necrosis factor. 
regulated in an age- and gender-dependent manner and is upregulated in individuals who smoke ${ }^{78}$.

In summary, age, gender and genetic background will have to be integrated into conventional SARS-CoV-2 assays and COVID-19 models to facilitate the screening of antiviral drugs and antibodies and predict therapeutic responses. Conventional COVID-19 assays can be classified into three categories: in vitro biochemical, pseudotyped and live virus assays (Fig. 2a). In this Review, we focus on cell culture models for COVID-19 research (Fig. 2b) and refer the reader to related reports and excellent reviews on conventional assays ${ }^{79-85}$.

\section{Cell culture models for COVID-19 research}

Theoretically, all cellular processes of the SARS-CoV-2 infection cycle could be used for assays to examine SARS-CoV-2 infectivity and for drug screens. At present, several experimental platforms and a

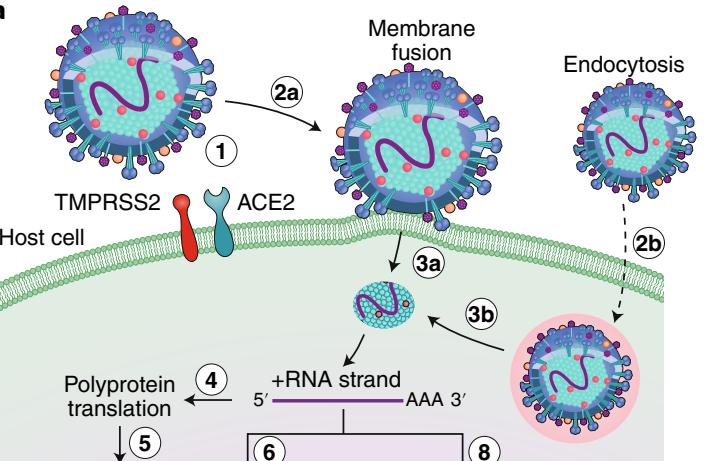

Processing -sgRNA

NSPs $S$-sgRNA UUU $3^{\prime}=$ RNA strand UUU $5^{\prime}$

$\downarrow$

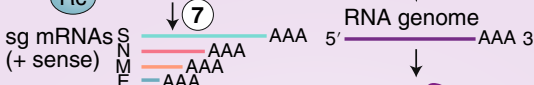
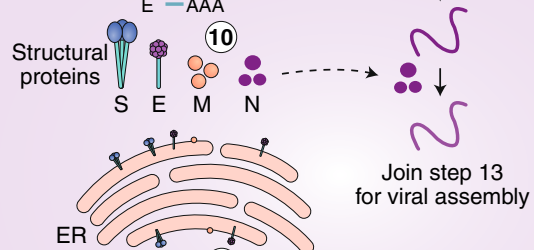

$\downarrow$ (11)

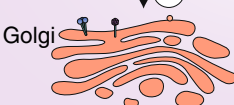

Budding, $\downarrow$ (12)

assembly
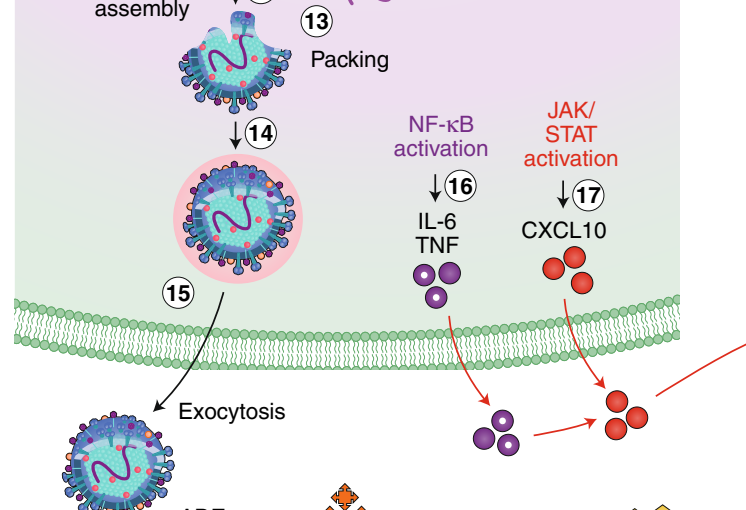

b

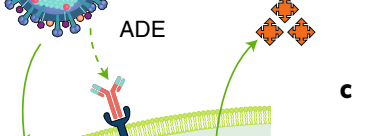

b

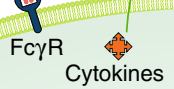

Viral antigen

$$
\stackrel{2}{2}
$$

APC (for example, macrophage)

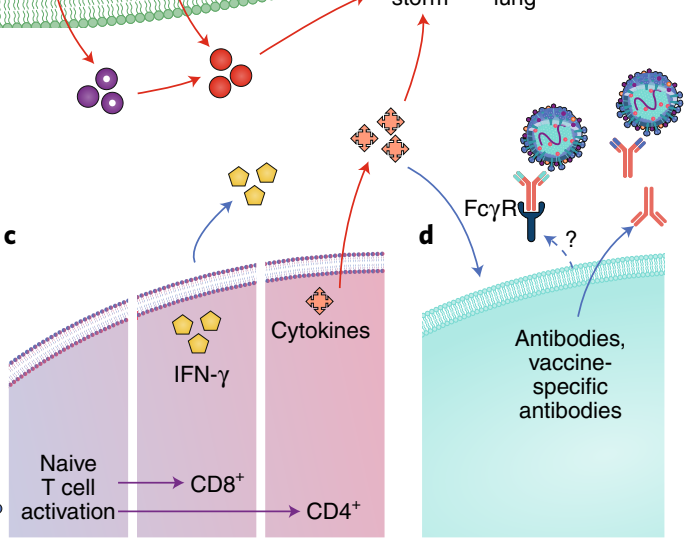

T cells

B cells

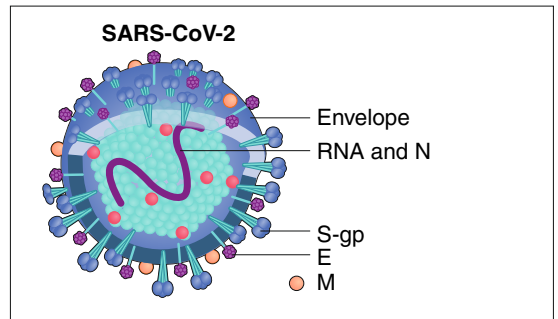

f Molecular interventions

1. Viral protein-host receptor interaction (camostat, chloroquine, nafamostat; monoclonal antibodies: 47D11, CR3022, S230, S304, S309,S315; rC-ACE2-Ig, vaccine: mRNA-1273,BNT162b2)

2a. Membrane fusion (EK1C4, nelfinavir)

2b. Endocytosis (Imatinib)

3a. Endosomal acidification (hydroxychloroquine)

5. Protein translation and proteolysis (disulfiram, lopinavir, ritonavir)

6. and 7. Subgenomic RNA transcription

8. and 9. RNA genome replication

(cyclosporin A, favipiravir, remdesivir)

10. Structural protein translation (NA)

13. Packaging (NA)

16. 17. Pro-inflammatory pathways

(baricitnib (JAKi), cyclosporin A

dexamethasone, tocilizumab (anti-IL-6R))

Antibodies, Chemicals, Human sera,

Recombinant proteins, Vaccines
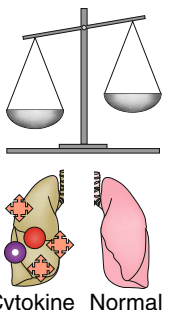

lung 


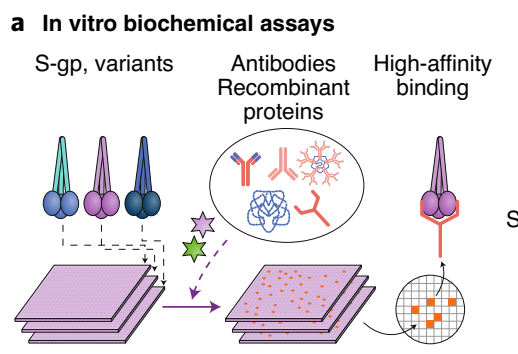

- Cell-free, quantitive and versatile - Enable rapid serological analysis - Adequate for antigen-antibody assays - Aid rational designs of inhibitors - Facilitate a molecular and structural assay - Need to validate these in vitro results

\section{b Animal models}

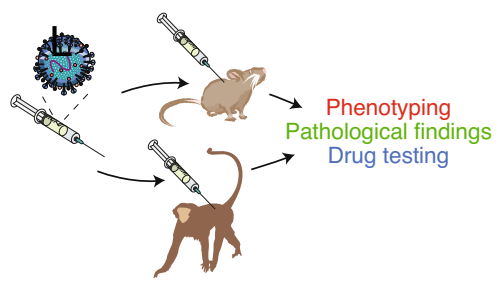

- Important for COVID-19 clinical studies - Recapitulate many clinical features - Offer clinically relevant strategies - Have species differences in drug response - Costly, restricted use of some animals - Require high biosafety levels

\section{Pseudotyped virus assays}

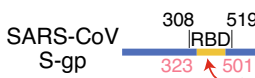

SARS-CoV-2, S-gp or RBD sequence VSV $\Delta \mathrm{G}+$

VSV $\Delta G+V S V-G$ SARS-CoV-S-gp chimera SARS-CoV-S-gp (pseudotypes) Measure

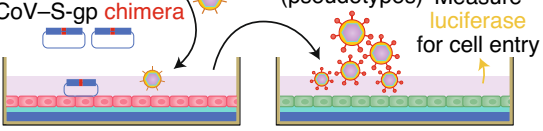

293T cells

Target cells

- Use an incompetent viral replicon - Safe for a broad host range

- Comparable to assays with wild-type

viruses

- With reporters for imaging and drug screens

- For infectivity and neutralization assays
2D ALI
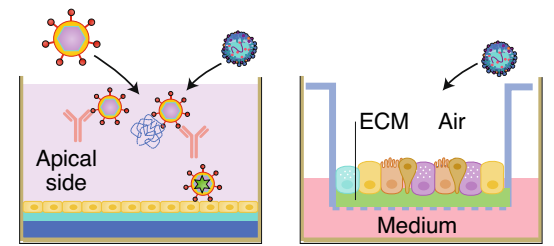

- Suitable for culturing various types of cells - Homogeneous growth, less variability - Commonly used for SARS-CoV-2 assays - Adaptable to high-content imaging

- Adjustable to ALI formats and drug screen

- Does not mimic 3D tissue niches in vivo
Live virus assays
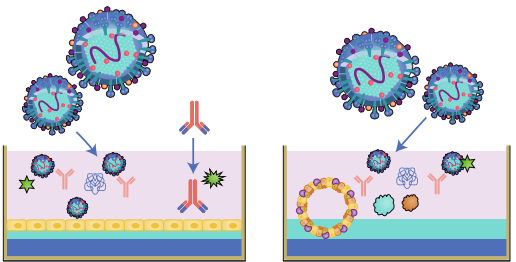

- Widely used as standard assays - For diverse types of cells expressing ACE2 - Assess drug and antibody efficacy - Suitable for viral cell-entry assays - Use SARS-CoV-2, highly contagious - Require high biosafety levels
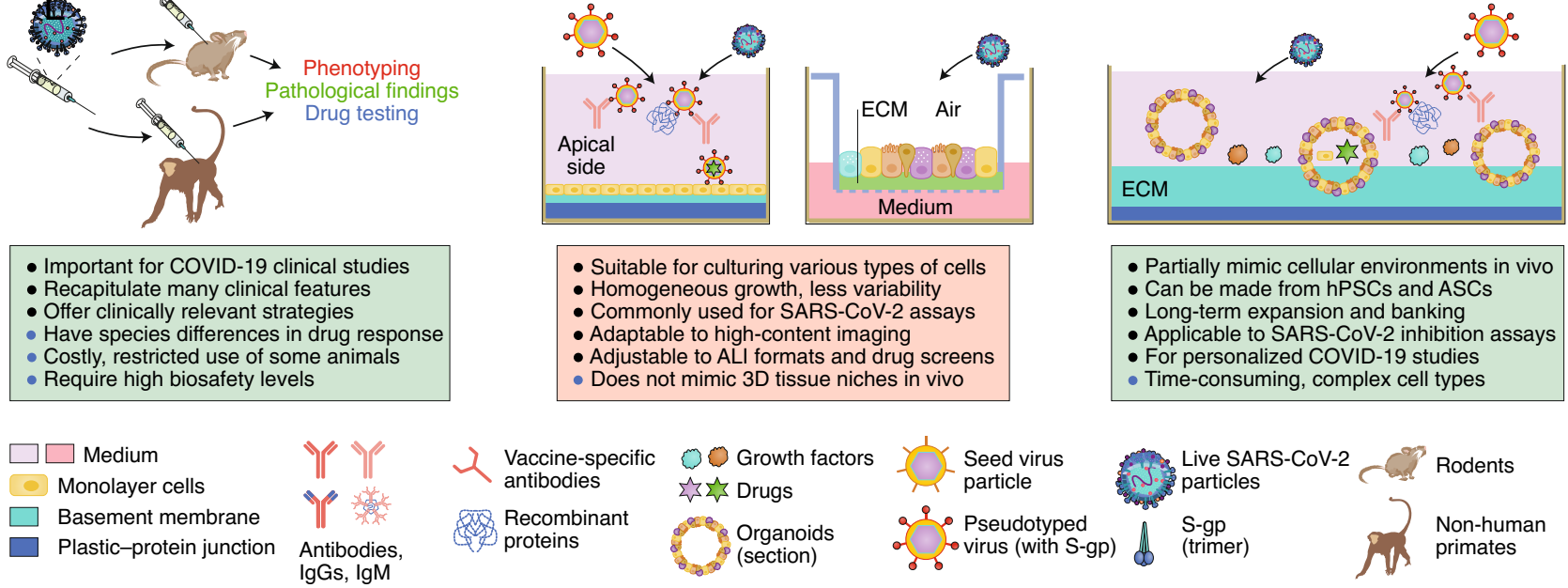

- Partially mimic cellular environments in vivo - Can be made from hPSCs and ASCs - Long-term expansion and banking - Applicable to SARS-CoV-2 inhibition assays - For personalized COVID-19 studies - Time-consuming, complex cell types

Fig. 2 | COVID-19-related assays. a, Assays are categorized as in vitro cell-free molecular and biochemical, pseudotyped virus and live virus assays. Pseudotyped virus experiments are exemplified by pseudotyped vesicular stomatitis virus (VSV) harbouring envelope glycoprotein (VSV-G) and SARS-CoV-S-gp chimeras. At $16 \mathrm{~h}$ after inoculation, the pseudotyped viral entry is analysed by determining luciferase activity in cell lysates ${ }^{86}$. VSV with deletion of the envelope glycoprotein (VSV $\Delta \mathrm{G}$ ) is used for normalization. b. Assays can be animal models, 2D monolayer cell culture, 2D ALI Transwell culture or 3D organoids. The combination of platforms empowers the utility of these assays for COVID-19 drug and vaccine development. ECM, extracellular matrix.

cell types exist for clinical and experimental coronavirus research, all of which have benefits and limitations (Fig. 2b). Here we focus on the three major systems used to study COVID-19:2D monolayer cell culture, adapted 2D air-liquid interface (ALI) methods, and 3D culture or organoids (Fig. 2b).

2D monolayer culture. 2D monolayer cell cultures (Fig. 2b) of various cell lines, such as 293 T, A549, BHK, Caco-2, MDBK, PK-15 and Vero cells (available from the American Type Culture Collection) have been used to investigate SARS-CoV-2 cell entry and for drug testing ${ }^{41,86}$. TMPRSS2-expressing Vero-E6 cells, which have a similar ACE2 structure to that of human cells, are highly susceptible to SARS-CoV-2 infection ${ }^{14,87}$ and represent an effective culture method to propagate SARS-CoV-2 and measure the viral load of SARS-CoV-2 variants.

ALI assays. ALI culture mimics the in vivo airway environment and is widely used to investigate the maturation and for functional assessment of the airway epithelium ${ }^{88}$. ALI assays enable the apical side of the epithelium to contact the air and the basolateral side to access the differentiation medium through a microporous membrane (Fig. 2b). Two-dimensional ALI is particularly suitable to evaluate links between related airborne lung disease pathologies and susceptibility to severe SARS-CoV-2 infection ${ }^{16}$. Limitations of this method are an inability to passage the culture, which means that it cannot be scaled up and used in high-throughput assays, and its inability to generate more complex tissue structures, such as alveoli. Historically, growth and differentiation of respiratory basal cells in an ALI culture has been challenging in the absence of non-basal cells. For example, KRT5-GFP ${ }^{+}$basal cells of the mouse trachea require a 500-fold excess of non-basal cells in ALI experiments to achieve approximately $6 \%$ colony-forming efficiency ${ }^{89}$ (based on counting large colonies) at day 21. Using an adapted 3D sphere-forming assay, Hogan and colleagues were able to seed single KRT5-GFP ${ }^{+}$basal cells of the mouse trachea in the absence of stroma or non-basal cells ${ }^{90}$. This $3 \mathrm{D}$ culture adaptation leads to a rapid formation of 'tracheospheres' within one week and a sphere-forming efficiency that is comparable to ALI experiments described above ${ }^{89,90}$.

3D cell culture and organoids. Unlike 2D cell culture, 3D cell culture is an artificially created platform that mimics the in vivo 
environment of living cells and tissues. Cells are usually grown in suspension in suitable medium or extracellular matrices (such as Matrigel and collagen) to form spheroids or 3D colonies. The extracellular matrix components and physical forces have a vital role in regulating cell behaviour. Current organoid protocols partially recapitulate $3 \mathrm{D}$ cellular environments in vivo and retain the genetic and epigenetic features of human cells. They can be expanded over a long period, banked for personalized medicine (Fig. 2b) and used to model viral infectious diseases ${ }^{6-9,91,92}$. Three-dimensional organoids can be dissociated and adapted to $2 \mathrm{D}$ ALI cultures to facilitate directed differentiation of airway stem or progenitor cells into mature cells for downstream assays ${ }^{93,94}$ and apical viral respiratory infection ${ }^{94}$. An overview of the strengths and limitations of 2D and 3D culture is presented in Fig. 2b and by previous Reviews ${ }^{6,8,95}$. Organoids thus represent a powerful platform for COVID-19 research.

\section{hPSC-derived organoids for COVID-19 research}

Organoids can be derived from human embryonic stem cells (ESCs) or human induced human pluripotent stem cells (hiPSCs) (here we use the term hPSC for both) and maintained as a $3 \mathrm{D}$ tissue that is capable of self-organizing and self-renewal in vitro. They have been used successfully for disease modelling and drug discovery ${ }^{6,7}$, thus paving the way for study of COVID-19 in vitro.

Modelling COVID-19. The first proof-of-concept experiment demonstrated that SARS-CoV-2 infects human blood-vessel and kidney organoids ${ }^{14}$, and this infection can be blocked with human recombinant $\mathrm{ACE} 2^{14}$. Subsequent reports confirmed that diverse types of hPSC-derived organoids, including intestinal, cardiac, brain, choroid plexus and lung organoids, can be used as disease models to study the tropism of SARS-CoV-2 and for drug screening $^{10,12,15,17,18}$. Lung organoids are particularly suitable, as epithelial cells of the respiratory airways and alveoli are both targets and effectors of SARS-CoV-2 infection (Fig. 3).

Lung organoids. The human lung is a complex organ with highly branched and progressively thinner tubes that carry air into the distal alveolar sacs. It comprises multiple integrated compartments: proximal and intermediate airways, respiratory bronchioles and alveoli ${ }^{96}$ (Fig. 3a,b). Each compartment is populated by various cell types, including epithelial, vascular, stromal and immune cells $^{78,97-99}$ (Fig. 3b,e). The intermediate airways have a pseudostratified epithelial layer that holds heterogeneous cell types, including secretory club cells, multiciliated cells, mucus-producing goblet cells, transient secretory cells and basal (stem) cells (Fig. 3b,e). The distal respiratory bronchioles are lined with a poorly characterized cuboidal epithelium. The alveoli are covered by alveolar epithelial type 1 and 2 cells (AECIs and AECIIs), which are important for gas exchange and alveolar homeostasis. Each compartment also has its own stem/progenitor population with specialized functions in response to environmental insults (Fig. 3b,e).

Airway epithelial cells are generated from hPSCs by imitating multi-stage lung developmental trajectories ${ }^{100}$-for instance, to derive lung bud organoids that recapitulate lung development and disease ${ }^{101,102}$. Lung organoids containing more mature epithelial cells have also been created from hPSCs in vitro ${ }^{10,17,103-106}$. The derivation of lung organoids varies from protocol to protocol. However, the major consensus steps may be summarized, on the basis of a well-documented protocol ${ }^{107}$, as follows. First, definitive endoderm is induced from hPSCs by activin. Second, anterior foregut endoderm and foregut spheroids are sequentially formed by inhibiting BMP 4 , TGF- $\beta$ and GSK3 $\beta$ in the presence of FGF4 and smoothened agonist. Third, bud-tip progenitor organoids are induced by FGF7, ATRA and GSK3 $\beta$ inhibition. Finally, complex lung organoids containing airway-like structures, mesenchymal-like cells and alveolar progenitors are obtained by prolonged incubation with foetal bovine serum and FGF10 (Fig. 3c).

Lung organoids are classified into bronchospheres, bronchioalveolar organoids and alveolospheres ${ }^{98,108}$ (Fig. 3d). In bronchospheres, secretory club cells and basal cells represent stem-like cells. Secretory club cells are a SARS-CoV-2 target, as they co-express the highest levels of ACE2 and TMPRSS2, compared with basal, ciliated and alveolar cells in the lung ${ }^{109}$ (Fig. 3f). ACE2, TMPRSS2 and FURIN are also co-expressed in bronchial transient secretory cells, which show high Rho GTPase activity and high levels of viral processes related to membrane remodelling or the immune system ${ }^{78}$, probably underlying their vulnerability to SARS-CoV-2 infection.

Alveolospheres contain flat AECIs and cuboidal AECIIs (Fig. 3d,e). AECIIs function as stem/progenitor cells in the adult lungs ${ }^{110}$, co-express ACE2 and TMPRSS2, and serve as a major SARS-CoV-2 target ${ }^{111,112}$. Not surprisingly, alveolar pneumocytes (including AECII cells) are severely affected in patients with COVID-19, leading to diffuse alveolar damage, respiratory failure and increased mortality ${ }^{39,40,113,114}$. For this reason, hPSC-derived lung organoids should be particularly useful for the study of severe COVID-19.

Drug discovery. hPSC-derived alveolar organoids have been used in SARS-CoV-2 infection assays, high-throughput drug screens and drug repurposing ${ }^{10,17,115-118}$. For example, the androgen receptor signalling inhibitors finasteride and dutasteride reduced the infectivity of SARS-CoV-2 in lung alveolar organoids derived from human ESCs by lowering levels of ACE2 and TMPRSS2 ${ }^{17}$. A high-throughput drug-repurposing screen in organoids identified multiple compounds (imatinib, mycophenolic acid and quinacrine dihydrochloride) that inhibit the cell entry of SARS-CoV- $2^{10}$. Similarly, an ACE2 blocking antibody inhibited viral entry in an organoid model, enhanced the activity of M2 macrophages and suppressed pro-inflammatory effects mediated by M1 macrophages ${ }^{118}$. These in vitro experiments confirm that alveolar precursors and differentiated AECIIs are permissive to SARS-CoV-2 infection, elicit a cytokine response and can be used to identify compounds that block SARS-CoV-2 infection.

Despite these promising initial results, organoid models also have a number of limitations that should be considered. For instance, hPSCs are prone to genomic instability in long-term in vitro culture ${ }^{119-123}$. Further, differences between protocols among different laboratories inevitably increase experimental variability, and cell culture and differentiation protocols are inherently time-consuming ${ }^{107,124}$. Finally, immature differentiation of lung organoids under suboptimal culture conditions remains a frequently encountered and unresolved issue ${ }^{125}$.

Human lung organoids often produce developmentally immature foetal lung tissues with a higher proliferation rate in vitro ${ }^{101,105,117,126}$. Epithelial cells from organoids derived from human ESCs express precursor markers such as NKX2.1 and SOX $9^{17}$. As a partial solution, 3D-organoid-converted 2D ALI cultures are increasingly used to enhance the maturity of differentiated respiratory epithelial cells for downstream analysis ${ }^{116,117,127}$. Nonetheless, a deeper understanding of the developmental principles underlying cell maturation and niche environments is necessary to optimize organoid protocols. These insights could facilitate the creation of chemically defined media and improved extracellular matrices or scaffolds ${ }^{124,128,129}$.

In summary, hPSC-based organoids are valuable for personalized medicine and disease modelling. They provide excellent platforms for drug efficacy and drug-repurposing studies ${ }^{10,17,115}$. The expression of multiple SARS-CoV-2 susceptible genes in lung organoids makes them ideal models to study infectivity. However, we recommend verifying the results obtained from hPSC-derived organoids in animal models and organoids established from human ASCs. 
a

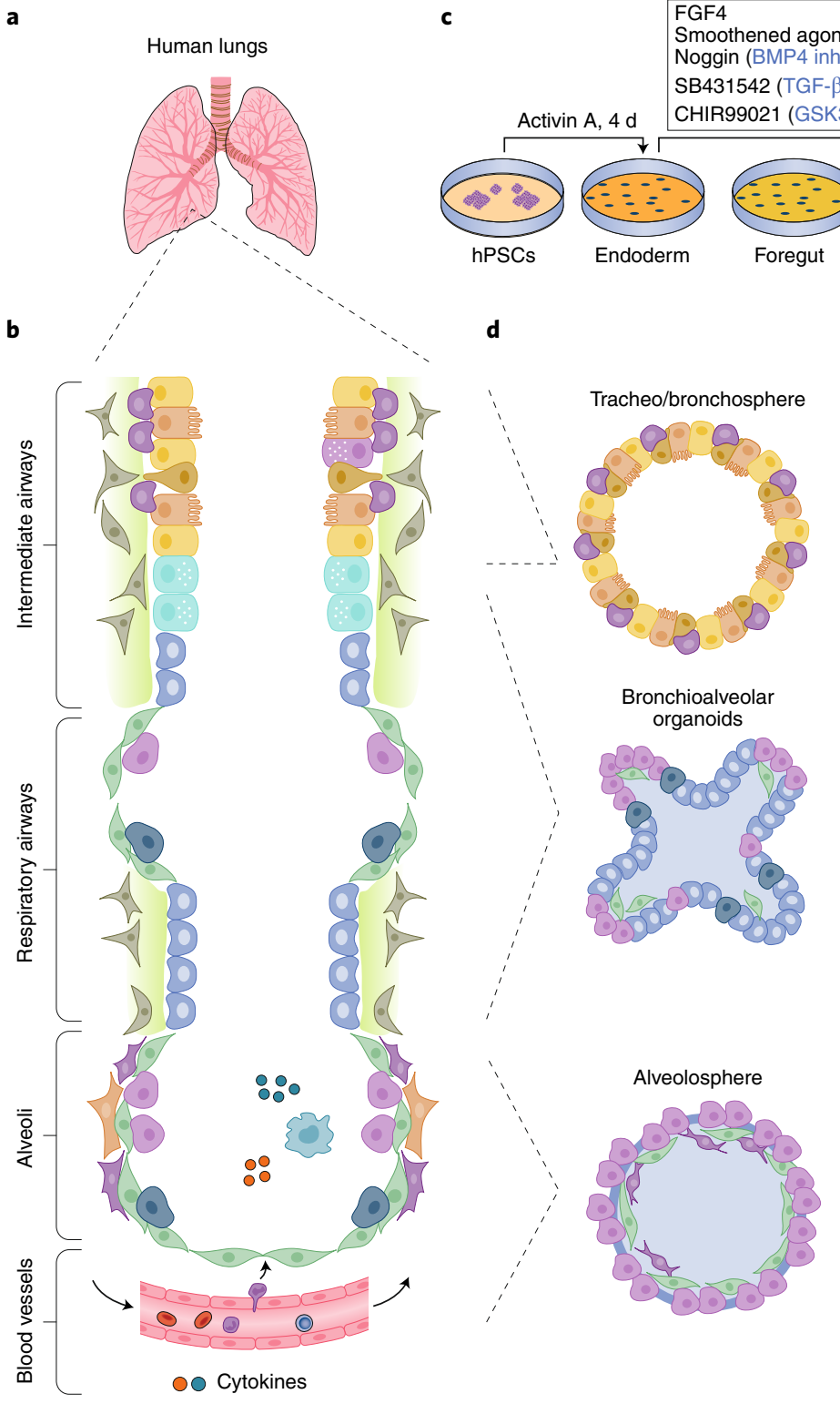

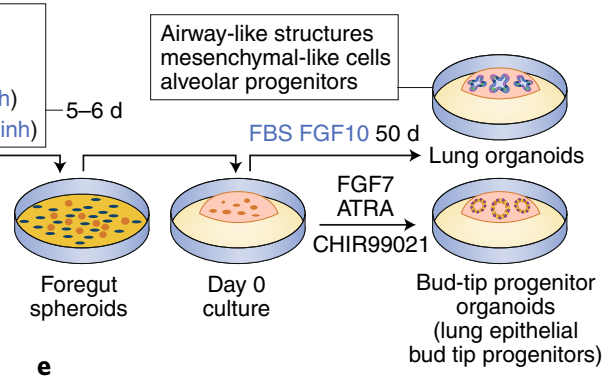

e

$$
\text { (lung epithelial }
$$

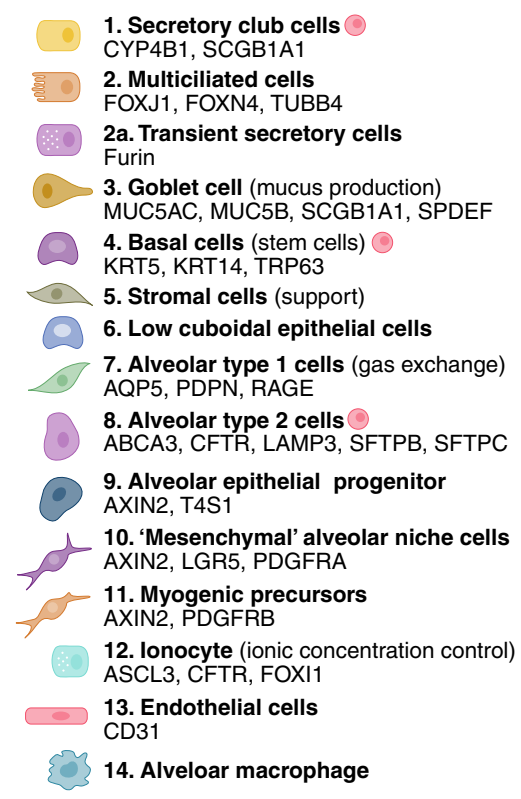

Stem/progenitor cells

f

Fig. 3 | Lung cell types and organoids. a, Human lung anatomy. b, Major cell types in different compartments of the human lung, partially adapted from ${ }^{78,97-99}$. c, A representative protocol for the generation of lung organoids containing cell types of interest ${ }^{107}$. $\mathbf{d}$, Schematic of lung organoids that model different cellular compartments of the lung. $\mathbf{e}$, Cell types in $\mathbf{b}, \mathbf{d}$, with gene and protein markers listed alphabetically $78,97-99,163,164$. $\mathbf{f}$, Representative single-cell RNA-sequencing analysis of SARS-CoV-2 receptor gene expression and co-expression ${ }^{109}$ (co-exp) in major cell types of the respiratory airways and alveoli. Nasal secretory cells are used as control for comparison. The size of the dots is proportional to the percentage of cells that express indicated genes (adapted from data in ref. ${ }^{109}$ ). ABCA3, ATP-binding cassette subfamily A member 3; AQP5, aquaporin 5; ASCL3, achaete-scute family BHLH transcription factor 3; ATRA, all-trans retinoic acid; BMP4, bone morphogenetic protein 4; CFTR, cystic fibrosis transmembrane conductance regulator; CYP4B1, cytochrome P450 family 4 subfamily B member 1; FBS, foetal bovine serum; FOXI1, forkhead box I1; FOXJ1, forkhead box J1; FOXN4, forkhead

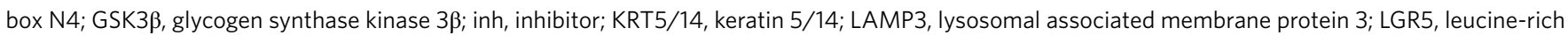
repeat-containing G-protein coupled receptor 5; PDGFRA/B, platelet-derived growth factor receptor- $\alpha / \beta$; PDPN, podoplanin; SCGB1A1, secretoglobin family $1 \mathrm{~A}$ member 1 ; SFTPB/C, surfactant protein B/C; SPDEF, SAM pointed domain containing ETS transcription factor; TUBB4, tubulin- $\beta$ 4B class IVb.

\section{ASC-derived organoids for COVID-19 research}

The definition of ASCs varies in the scientific literature due to the complexity of cellular properties, including cellular dynamics ${ }^{130}$, heterogeneity ${ }^{131}$ and plasticity ${ }^{132}$. In addition, it can be difficult to distinguish ASCs from progenitor cells. In this Review, ASCs are defined as rare, mostly quiescent, and multipotent cells found in adult tissues. They are capable of long-term self-renewal, generate intermediate cell types (progenitors) with limited self-renewal potential, and differentiate into tissue-specific cells ${ }^{7,97}$. ASCs can be isolated from the adult issue and maintained in cell culture indefinitely if supplemented with appropriate microenvironments and growth factors. ASCs and progenitors serve as valuable alternatives to hPSCs, providing a source of fully mature cells for functional analysis.

Intestinal and nasal organoids. Intestinal organoids and nasal spheroids have been derived from donor biopsies and were previously used to predict drug responses in patients with cystic fibrosis ${ }^{95}$. Differentiated enterocytes express ACE2 and TMPRSS2 (Fig. 4a) and substantial titres of SARS-CoV-2 particles have also been detected 

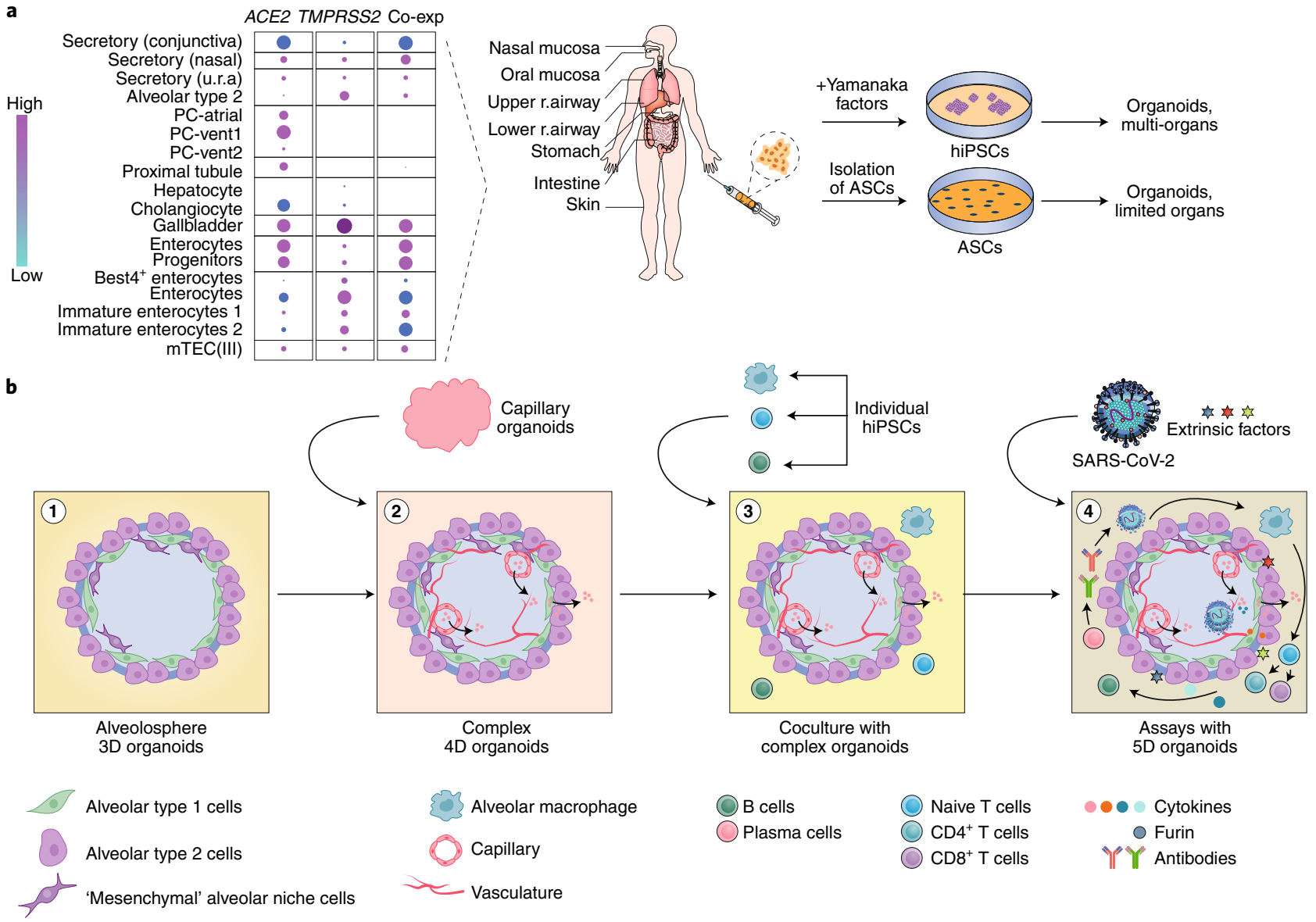

Fig. 4 | Stem cell-based organoids to assess SARS-CoV-2 susceptibility. a, SARS-CoV-2 receptor gene expression and co-expression in human cells (adapted from ref. ${ }^{109}$ ). Isogenic organoids can be generated from ASCs and hiPSCs (right panel). The size of the dots is proportional to the percentage of cells that express the indicated gene. $\mathbf{b}$, Development of multi-dimensional organoids to model the complexity of immunological and hyperinflammatory complications in patients with COVID-19. Abbreviations: mTEC (III), medullary thymic epithelial cells of the foetal thymus; PC-atrial, pericytes in the atrium of the heart; PC-vent, pericytes in the ventricle of the heart; r., respiratory; secretory (u.r.a), secretory cells from the upper respiratory airway.

in enterocytes of intestinal organoids ${ }^{13}$. Transcriptomic analysis indicated a strong viral response with enrichment of CXCL10 and CXCL11 mRNAs ${ }^{13}$, closely related to a cytokine storm. This study supports the use of ASC organoids to study SARS-CoV-2 pathophysiology in vitro.

Interestingly, the nasal mucosa also co-expresses high levels of ACE2 and TMPRSS2 ${ }^{109}$ (Fig. 4a), consistent with the heavy SARS-CoV-2 particle load in the nasal cavity of patients with COVID-19. The nasal mucosa has a similar epithelial lining to that of the upper respiratory airway, including secretory club cells and basal stem cells ${ }^{109}$. As nasal biopsies are minimally invasive compared with intestinal or lung biopsies, nasal spheroids provide a valuable resource and surrogate for lung organoids.

Lung organoids. Evidence suggests that both ASC-like cells and progenitors exist in different compartments of the lungs. Basal cells in the intermediate airways meet the definition of generic $\mathrm{ASCs}^{90,94}$. Basal stem cell organoids contain basal cells, secretory goblet cells and ciliated cells (Fig. 3d,e). Airway basal stem cells have been isolated from human biopsies and expanded for functional assays of the airway repair response after SARS-CoV-2 infection ${ }^{16}$.

ASC-like cells or progenitors have also been found within the SCGB1A1 ${ }^{+}$secretory club and AXIN2+ AECII cell populations in human adult lungs ${ }^{96,108}$ (Fig. 3b,e). Mouse genetic-lineage analysis revealed that surfactant protein C (SFTPC)-positive AECII cells in the alveolar niche are ASC-like cells, and give rise to self-renewing 'alveolospheres' that contain both AECII and AECI-like cells ${ }^{110}$. In mice, rare Axin2 ${ }^{+}$AECIIs also act as alveolar stem cells and secrete Wnt molecules to recruit 'bulk' AECIIs as the progenitors $^{133}$. A distinct population of mouse IL1R1 ${ }^{+}$AECIIs can become damage-associated transient progenitors, which then differentiate into mature $\mathrm{AECIs}^{134}$. In mouse and human lungs, similar alveolar epithelial progenitors reside within the AECII pool and generate mature AECIs and AECIIs from alveolar organoids ${ }^{135}$. Thus, AECIIs constitute an important stem/progenitor source in the alveoli.

Human alveolar organoids have been derived from adult AECIIs to assess SARS-CoV-2 infection ${ }^{11,112,127,136,137}$. These in vitro experiments confirm that AECIIs are the principal target of SARS-CoV-2. SARS-CoV-2-infected alveolar organoids mirror many features of patients with COVID-19, including cytokine release, IFN and immune response, loss of surfactant proteins, and cell death. AECII-based organoids, derived in a feeder-free and chemically defined culture system, could be sustained long-term ${ }^{112}$ and revealed that entry of few $(\geq 1)$ SARS-CoV-2 particles into alveolar cells can lead to a full infection. Genes associated with cell death, cell adhesion, and surfactant proteins were also upregulated in SARS-CoV2-infected AECIIs ${ }^{112}$.

IFN-mediated inflammatory signalling is a typical response to the SARS-CoV-2 infection documented in these studies. An increase in the IFN response was associated with a lower SARS-CoV-2 burden 
(around $60 \mathrm{~h}$ after SARS-CoV-2 infection of alveolar organoids) and vice versa for a decrease in the IFN response ${ }^{112}$. Pretreatment of alveolar organoids with low dose IFN- $\alpha$ and IFN- $\gamma$ reduced SARS-CoV-2 replication ${ }^{11}$. By contrast, IFN inhibition endorsed viral replication ${ }^{11}$. Pretreatment of alveolar organoids with IFN- $\beta$ also reduced expression of the viral RNA gene $N$, which encodes the SARS-CoV-2 nucleoprotein ${ }^{127}$. These findings suggest that the administration of IFNs may be a possible prophylactic measure against severe SARS-CoV-2 infection.

Pharmacological inhibition of SARS-CoV-2 infection with small molecules is of considerable interest. To our knowledge, ASC-derived lung organoids have not yet been used for drug-repurposing or drug-discovery studies. However, a study confirmed that remdesivir decreases SARS-CoV-2 $N$ gene expression more effectively than IFN- $\beta$ or hydroxychloroquine in infected alveolar organoids ${ }^{127}$. This finding is intriguing but contradicts the ineffectiveness of remdesivir in the recent clinical trial discussed above ${ }^{48}$. Inconsistencies between the results from alveolar organoids and the clinical trial need to be further investigated.

In summary, lung organoids derived from adult human lungs generate respiratory epithelial cells with high maturity compared to hPSC-derived organoids and are suitable for studying COVID19. However, it is often difficult to obtain lung tissues with the desired quality and materials are scarce, as samples are typically acquired from bronchioalveolar washings and lung explants with institutional review board approval ${ }^{108}$. In contrast to hPSC-derived lung organoids, ASC- or progenitor-based lung organoids exhibit limited self-renewal capacity, usually less than five passages. Developmental paradigms for hPSC-derived lung organoids can guide the derivation of long-term expandable lung organoids from the adult lung ${ }^{94}$. For instance, FGF7 and FGF10 are vital in establishing long-term expandable lung organoids from adult tissues $^{94,138}$. Interestingly, both factors are also required in the final steps to generate hPSC-derived lung organoids ${ }^{107}$ (Fig. 3c).

Improving lung regeneration. Little is known about the regenerative capacity of the alveolus in COVID-19 patients. Organoid studies revealed that SARS-CoV-2-infected AECIIs exhibit defence and repair mechanisms to combat injury, such as cytokine secretion, resistance to apoptosis and cellular senescence ${ }^{11,112,118,139}$. AECIIs still proliferate, transit to different cellular states and differentiate into AECI-like cells. These cellular processes closely mimic regenerative responses in mouse and human injury models ${ }^{110,133-135}$. Further support for a targeted regenerative response comes from a study demonstrating that AECIIs proliferate and differentiate into squamous AECIs in severely affected alveoli of patients with COVID- $19^{40}$.

Despite the existence of endogenous repair mechanisms, a number of individuals who have recovered from COVID-19 will require therapy to restore the lost lung function and repair the damage to alveolar cells. Replacing damaged alveolar cells with suitably sourced AECIIs might be a possible way to improve lung function. Encouragingly, mature AECIIs of both mouse and human origins can be transplanted into injured mouse lungs ${ }^{140,141}$. Vunjak-Novakovic and colleagues ${ }^{142}$ proposed an airway-specific method to de-epithelialize the distal lung airways and preserve the basement membrane and vascular endothelium. This approach enabled the functional vascularization of lung grafts to support the attachment and growth of hiPSC-derived epithelial cells in a rat model $^{142}$. Similar transplantation approaches using organoid-derived lung epithelial cells may be applicable for treating COVID-19 patients with the severe epithelial injury in the future. Still, many preclinical challenges remain to be overcome, most notably relating to source cell identity, immunological compatibility and functional integration into the host.

\section{Opportunities and challenges}

SARS-CoV-2 infection does not only affect the lung but can damage any cell that expresses ACE2 or co-expresses ACE2 and TMPRSS2 (Fig. 4a). The ACE2 receptor, initially identified as a cardiac regulator, is present on oral mucosa, AECII pneumocytes, intestinal, kidney, cardiac, smooth muscle and endothelial cells ${ }^{143-146}$. Transcriptomic profiling provides a comprehensive view of ACE2 and TMPRSS2 expression in cells of the human body ${ }^{109}$. These datasets are particularly helpful when choosing specific organoids for COVID-19 research (Fig. 4a).

Established cell lines and organoids. Currently, hiPSC-derived patient-specific lung organoids recapitulate the pathophysiology of various lung diseases, such as surfactant deficiency ${ }^{147}$, cystic fibrosis ${ }^{106}$, Hermansky-Pudlak syndrome $e^{101,148}$ and respiratory syncytial and parainfluenza virus infection ${ }^{101,102}$. In another example, AECIIs derived from a child with a lethal neonatal respiratory distress syndrome caused by a homozygous SFTPB mutation mimicked aspects of this syndrome, including the deficiency in surfactant processing ${ }^{147}$. The abnormal processing could be restored in gene-corrected AECIIs from this individual ${ }^{147}$. So far, experiments with hiPSC-derived organoids have confirmed that lung epithelial cells are susceptible to SARS-CoV-2 infection, express SARS-CoV-2 host factors, and provoke an intrinsic epithelial inflammatory res ponse $^{116,117,126}$. However, these hiPSC-derived lung organoids have not yet been used to reveal the differences associated with inherent variations to infection and immune activation in large cohorts of patients with COVID-19116,117,126. Organoids derived from established hiPSC lines and cell banks from individual donors or patient biopsies provide a valuable and convenient tool to assess risk factors and therapeutic outcomes in the most vulnerable populations and implement targeted prevention at low cost. Currently, lung, cardiac, intestinal, liver, kidney and capillary organoids are immediately available to serve these purposes.

Complex organoid assays. One major remaining challenge in the application of organoids is the lack of complexity and extensive intercellular interactions compared to the in vivo situation. Complex lung airway organoids can be derived by integrating human adult primary bronchial epithelial cells and lung fibroblasts with lung microvascular endothelial cells to study disease-relevant cell-cell interactions ${ }^{149}$. However, hPSC-derived alveolospheres typically contain few cell types (for instance AECIs and AECIIs) and do not include adjacent capillaries composed of endothelial cells (Fig. 3b).

The endothelium is connected with alveolar cells by the basement membrane and acts as an alveolar niche. Mouse alveolospheres form more easily in organoid co-cultures with lung endothelial cells ${ }^{150}$. Successful alveolar repair requires restoration of the spatial relationship between alveolar cells and the endothelium. Endothelial cells can receive reparative signals from AECIs after acute injury ${ }^{151}$ and enhance alveologenesis through diverse signalling factors such as endothelial-derived angiocrine factors and platelet-derived SDF$1^{152,153}$. Endothelial cells also secrete the vascular endothelial growth factor, monocyte chemoattractant protein-1, IL-6 and IL-8, all of which aggravate the cytokine storm ${ }^{35}$. Reduced vascular endothelial cadherin expression on endothelial cells ${ }^{154}$ increases vascular permeability and pulmonary dysfunction in acute respiratory distress syndrome. For these reasons, vascularised lung organoids should provide valuable insights into SARS-CoV-2-mediated lung damage and repair.

So far, the generation of vascularised lung organoids has not been achieved, although vascularization occurs in lung organoids grafted into animals to promote cell differentiation ${ }^{101,129}$. For the liver, vascularised organoids termed liver buds have been reported, which consist of hiPSC-derived hepatic endoderm, endothelial cells and bone marrow stromal cells ${ }^{155}$. Similarly, the integration 
of vasculature into cerebral organoids seems to accelerate functional maturation of neurons ${ }^{156}$. It seems likely that vascularised lung organoids might exhibit comparable properties. At any rate, improved protocols for complex organoids with integrated capillary systems are expected to more accurately recapitulate the alveolar SARS-CoV-2 response (Fig. 4b).

Another major challenge is the lack of host factors, immune cells and inflammatory responses in existing organoid assays ${ }^{66,157}$. Drug and antibody effects observed in these assays may not reflect genuine responses of patients with COVID-19, who often have additional medical conditions such as cardiovascular disorders or diabetes that alter cellular behaviour ${ }^{158}$. Ideally, organoids should include multiple cell types to closely mimic in vivo environments and the complexity of hyperinflammatory complications in patients (Fig. 4b). We previously proposed a concept that encompasses the multi-dimensionality of organoids ${ }^{6}$. In addition to $3 \mathrm{D}$ structures, we propose that $4 \mathrm{D}$ organoids emphasize the developmental time scale in an organoid culture. By comparison, 5D organoids would further integrate extrinsic factors to simulate host environmental, immunological and inflammatory signals, which are absent from current organoid cultures ${ }^{6}$. Highly sensitive ELISA-based analysis of the cell culture medium is essential for monitoring cytokine storm inhibition in these complex organoids.

In the future, organoids could also have a role in vaccine development. Traditional vaccine development is a long-lasting process. Exploratory efforts on vaccine design, preclinical evaluation in animal models and clinical phase I, II, and III trials can take 15 years or more ${ }^{159}$. Although organoids may help identify potential molecular targets for vaccine design, they cannot be directly used to derive a vaccine due to the absence of the host immune system. However, organoid assays may provide valuable information about intermolecular interactions between viral proteins and host receptors and determine the efficacy of neutralizing antibodies from vaccinated individuals (Fig. 2). In this way, organoid platforms might accelerate vaccine development at all stages that involve exploratory work, preclinical evaluation and the assessment of efficacy in clinical trials. Encouragingly, the generation of immune cell organoids (for instance, $\mathrm{T}$ cell and $\mathrm{B}$ cell specific organoids) is feasible from hiPSCs ${ }^{160}$. The presence of T cells, B cells and macrophages in organoid systems might well improve vaccine assessment in the future (Fig. 4b).

Importantly, the integration of multiple cell types is limited by developmental constraints for a specific organ in a defined niche environment. As with all other organoid systems, a thorough understanding of the underlying developmental biology will be required for further progress in the creation of complex organoids.

\section{Concluding remarks}

The inherent physiological variability of human populations poses a major challenge for the assessment of individual susceptibility and therapeutic outcomes. The versatility of hPSC-based and ASC-based organoids makes them a useful platform to compensate for the shortcomings of current assays. Multi-tissue isogenic organoids from individual donors and patients enable robust molecular assessment of the vulnerability of individual patients and possibly predict therapeutic responses in patients with severe COVID-19. We envision that the combination of current assays with complex organoids will continue to improve COVID-19 research and treatment, and provide valuable lessons for the study of other viral diseases as well.

Received: 5 December 2020; Accepted: 22 June 2021; Published online: 2 August 2021

\section{References}

1. WHO Coronavirus (COVID-19) Dashboard. World Health Organization https://covid19.who.int/ (2021)
2. Yan, Y. et al. The first 75 days of novel coronavirus (SARS-CoV-2) outbreak: recent advances, prevention, and treatment. Int. J. Environ. Res. Pub. Health 17, https://doi.org/10.3390/ijerph17072323 (2020).

3. COVID-19 Clinical Trials. National Institutes of Health https://covid19.nih. gov/treatments-and-vaccines/clinical-trials (2021).

4. $\mathrm{Hu}$, J., Li, C., Wang, S., Li, T. \& Zhang, H. Genetic variants are identified to increase risk of COVID-19 related mortality from UK Biobank data. Preprint at medRxiv https://doi.org/10.1101/2020.11.05.20226761 (2020).

5. Pairo-Castineira, E. et al. Genetic mechanisms of critical illness in COVID-19. Nature 591, 92-98 (2021).

6. Chen, K. G. et al. Pluripotent stem cell platforms for drug discovery. Trends Mol. Med. 24, 805-820 (2018)

7. Clevers, H. Modeling development and disease with organoids. Cell 165, 1586-1597 (2016).

8. Fatehullah, A., Tan, S. H. \& Barker, N. Organoids as an in vitro model of human development and disease. Nat. Cell Biol. 18, 246-254 (2016).

9. Lancaster, M. A. \& Knoblich, J. A. Organogenesis in a dish: modeling development and disease using organoid technologies. Science 345, 1247125 (2014).

10. Han, Y. et al. Identification of SARS-CoV-2 inhibitors using lung and colonic organoids. Nature 589, 270-275 (2021).

11. Katsura, H. et al. Human lung stem cell-based alveolospheres provide insights into SARS-CoV-2-mediated interferon responses and pneumocyte dysfunction. Cell Stem Cell 27, 890-904 (2020).

12. Jacob, F. et al. Human pluripotent stem cell-derived neural cells and brain organoids reveal SARS-CoV-2 neurotropism predominates in choroid plexus epithelium. Cell Stem Cell 27, 937-950 (2020).

13. Lamers, M. M. et al. SARS-CoV-2 productively infects human gut enterocytes. Science 369, 50-54 (2020).

14. Monteil, V. et al. Inhibition of SARS-CoV-2 infections in engineered human tissues using clinical-grade soluble human ACE2. Cell 181, 905-913 (2020).

15. Pellegrini, L. et al. SARS-CoV-2 infects the brain choroid plexus and disrupts the blood-CSF barrier in human brain organoids. Cell Stem Cell 27, 951-961 (2020).

16. Purkayastha, A. et al. Direct exposure to SARS-CoV-2 and cigarette smoke increases infection severity and alters the stem cell-derived airway repair response. Cell Stem Cell 27, 869-875 (2020).

17. Samuel, R. M. et al. Androgen signaling regulates SARS-CoV-2 receptor levels and is associated with severe COVID-19 symptoms in men. Cell Stem Cell 27, 876-889 (2020).

18. Yang, L. et al. A human pluripotent stem cell-based platform to study SARS-CoV-2 tropism and model virus infection in human cells and organoids. Cell Stem Cell 27, 125-136 (2020).

19. Zhao, B. et al. Recapitulation of SARS-CoV-2 infection and cholangiocyte damage with human liver ductal organoids. Protein Cell 11, 771-775 (2020).

20. Hao, P., Zhong, W., Song, S., Fan, S. \& Li, X. Is SARS-CoV-2 originated from laboratory? A rebuttal to the claim of formation via laboratory recombination. Emerg. Microbes Infect. 9, 545-547 (2020).

21. Liu, S. L., Saif, L. J., Weiss, S. R. \& Su, L. No credible evidence supporting claims of the laboratory engineering of SARS-CoV-2. Emerg. Microbes Infect. 9, 505-507 (2020).

22. Andersen, K. G., Rambaut, A., Lipkin, W. I., Holmes, E. C. \& Garry, R. F. The proximal origin of SARS-CoV-2. Nat. Med. 26, 450-452 (2020).

23. Zhang, Y. Z. \& Holmes, E. C. A genomic perspective on the origin and emergence of SARS-CoV-2. Cell 181, 223-227 (2020).

24. Shen, Z. et al. Genomic diversity of severe acute respiratory syndrome-coronavirus 2 in patients with coronavirus disease 2019. Clin. Infect. Dis. 71, 713-720 (2020).

25. Wen, F. et al. Identification of the hyper-variable genomic hotspot for the novel coronavirus SARS-CoV-2. J. Infect. 80, 671-693 (2020).

26. Wertheim, J. O. A glimpse into the origins of genetic diversity in the severe acute respiratory syndrome coronavirus 2. Clin. Infect. Dis. 71, 721-722 (2020).

27. van Doremalen, N. et al. Aerosol and surface stability of SARS-CoV-2 as compared with SARS-CoV-1. N. Engl. J. Med. 382, 1564-1567 (2020).

28. Srinivasan, S. et al. Structural genomics of SARS-CoV-2 indicates evolutionary conserved functional regions of viral proteins. Viruses 12, https://doi.org/10.3390/v12040360 (2020).

29. Wang, C. et al. The establishment of reference sequence for SARS-CoV-2 and variation analysis. J. Med Virol. 92, 667-674 (2020).

30. Cui, J., Li, F. \& Shi, Z. L. Origin and evolution of pathogenic coronaviruses. Nat. Rev. Microbiol. 17, 181-192 (2019).

31. V'Kovski, P., Kratzel, A., Steiner, S., Stalder, H. \& Thiel, V. Coronavirus biology and replication: implications for SARS-CoV-2. Nat. Rev. Microbiol. 19, 155-170 (2021).

32. Hu, B., Guo, H., Zhou, P. \& Shi, Z. L. Characteristics of SARS-CoV-2 and COVID-19. Nat. Rev. Microbiol. 19, 141-154 (2021).

33. Zou, L. et al. SARS-CoV-2 viral load in upper respiratory specimens of infected patients. N. Engl. J. Med. 382, 1177-1179 (2020) 
34. Li, G. et al. Coronavirus infections and immune responses. J. Med Virol. 92 424-432 (2020).

35. Moore, J. B. \& June, C. H. Cytokine release syndrome in severe COVID-19. Science 368, 473-474 (2020).

36. Wang, W. et al. Detection of SARS-CoV-2 in different types of clinical specimens. JAMA 323, 1843-1844 (2020).

37. Pan, Y., Zhang, D., Yang, P., Poon, L. L. M. \& Wang, Q. Viral load of SARS-CoV-2 in clinical samples. Lancet Infect. Dis. 20, 411-412 (2020).

38. Fajgenbaum, D. C. \& June, C. H. Cytokine storm. N. Engl. J. Med. 383, 2255-2273 (2020).

39. Berlin, D. A., Gulick, R. M. \& Martinez, F. J. Severe Covid-19. N. Engl. J. Med. 383, 2451-2460 (2020).

40. Chen, J., Wu, H., Yu, Y. \& Tang, N. Pulmonary alveolar regeneration in adult COVID-19 patients. Cell Res. 30, 708-710 (2020)

41. Hoffmann, M. et al. SARS-CoV-2 cell entry depends on ACE2 and TMPRSS2 and is blocked by a clinically proven protease inhibitor. Cell 181, 271-280 (2020).

42. Liu, J. et al. Hydroxychloroquine, a less toxic derivative of chloroquine, is effective in inhibiting SARS-CoV-2 infection in vitro. Cell Disco. 6, 16 (2020).

43. Mehra, M. R., Desai, S. S., Ruschitzka, F. \& Patel, A. N. Retracted: Hydroxychloroquine or chloroquine with or without a macrolide for treatment of COVID-19: a multinational registry analysis. Lancet https://doi.org/10.1016/S0140-6736(20)31180-6 (2020).

44. Reina, J. Remdesivir, the antiviral hope against SARS-CoV-2. Rev. Esp. Quimioter. 33, 176-179 (2020)

45. Amanat, F. \& Krammer, F. SARS-CoV-2 vaccines: status report. Immunity 52, 583-589 (2020)

46. Caly, L., Druce, J. D., Catton, M. G., Jans, D. A. \& Wagstaff, K. M. The FDA-approved drug ivermectin inhibits the replication of SARS-CoV-2 in vitro. Antivir. Res 178, 104787 (2020).

47. Horby, P. et al. Dexamethasone in hospitalized patients with Covid-19. N. Engl. J. Med. 384, 693-704 (2021).

48. Pan, H. et al. Repurposed antiviral drugs for Covid-19-interim WHO solidarity trial results. N. Engl. J. Med. 384, 497-511 (2021).

49. ter Meulen, J. et al. Human monoclonal antibody combination against SARS coronavirus: synergy and coverage of escape mutants. PLoS Med. 3, e237 (2006).

50. Tian, X. et al. Potent binding of 2019 novel coronavirus spike protein by a SARS coronavirus-specific human monoclonal antibody. Emerg. Microbes Infect. 9, 382-385 (2020).

51. Yuan, M. et al. A highly conserved cryptic epitope in the receptor binding domains of SARS-CoV-2 and SARS-CoV. Science 368, 630-633 (2020).

52. Inoue, Y. et al. Clathrin-dependent entry of severe acute respiratory syndrome coronavirus into target cells expressing ACE2 with the cytoplasmic tail deleted. J. Virol. 81, 8722-8729 (2007).

53. Nomura, R. et al. Human coronavirus $229 \mathrm{E}$ binds to CD13 in rafts and enters the cell through caveolae. J. Virol. 78, 8701-8708 (2004).

54. Van Hamme, E., Dewerchin, H. L., Cornelissen, E., Verhasselt, B. \& Nauwynck, H. J. Clathrin- and caveolae-independent entry of feline infectious peritonitis virus in monocytes depends on dynamin. J. Gen. Virol. 89, 2147-2156 (2008).

55. FDA Approves First Treatment for COVID-19 (US Food \& Drug Administration, 2020); https://www.fda.gov/news-events/ press-announcements/fda-approves-first-treatment-covid-19

56. Ryabkova, V. A., Churilov, L. P. \& Shoenfeld, Y. Influenza infection, SARS MERS and COVID-19: cytokine storm-the common denominator and the lessons to be learned. Clin. Immunol. 223, 108652 (2021).

57. Ye, Q., Wang, B. \& Mao, J. The pathogenesis and treatment of the 'cytokine storm' in COVID-19. J. Infect. 80, 607-613 (2020).

58. Efficacy of tocilizumab on patients with COVID-19. ClinicalTrials.gov https://www.clinicaltrials.gov/ct2/show/NCT04356937 (2020).

59. Guaraldi, G. et al. Tocilizumab in patients with severe COVID-19: a retrospective cohort study. Lancet Rheumatol. 2, e474-e484 (2020).

60. Stone, J. H. et al. Efficacy of tocilizumab in patients hospitalized with Covid-19. N. Engl. J. Med. 383, 2333-2344 (2020).

61. Sterne, J. A. C. et al. Association between administration of systemic corticosteroids and mortality among critically ill patients with COVID-19: a meta-analysis. JAMA 324, 1330-1341 (2020).

62. Pan, X. et al. Asymptomatic cases in a family cluster with SARS-CoV-2 infection. Lancet Infect. Dis. 20, 410-411 (2020).

63. Lai, C. C. et al. Asymptomatic carrier state, acute respiratory disease, and pneumonia due to severe acute respiratory syndrome coronavirus 2 (SARS-CoV-2): facts and myths. J. Microbiol Immunol. Infect. 53, 404-412 (2020).

64. Park, S. E. Epidemiology, virology, and clinical features of severe acute respiratory syndrome -coronavirus-2 (SARS-CoV-2; coronavirus disease-19). Clin. Exp. Pediatr. 63, 119-124 (2020).
65. Ling, Z. et al. Asymptomatic SARS-CoV-2 infected patients with persistent negative CT findings. Eur. J. Radiol. 126, 108956 (2020).

66. Rokni, M., Ghasemi, V. \& Tavakoli, Z. Immune responses and pathogenesis of SARS-CoV-2 during an outbreak in Iran: comparison with SARS and MERS. Rev. Med Virol. 30, e2107 (2020).

67. Cao, Q., Chen, Y. C., Chen, C. L. \& Chiu, C. H. SARS-CoV-2 infection in children: transmission dynamics and clinical characteristics. J. Formos. Med Assoc. 119, 670-673 (2020).

68. Chidini, G., Villa, C., Calderini, E., Marchisio, P. \& De Luca, D. SARS-CoV-2 infection in a pediatric department in Milan: a logistic rather than a clinical emergency. Pediatr. Infect. Dis. J. 39, e79-e80 (2020).

69. Lu, X. et al. SARS-CoV-2 infection in children. N. Engl. J. Med. 382, 1663-1665 (2020)

70. Wu, Z. \& McGoogan, J. M. Characteristics of and important lessons from the coronavirus disease 2019 (COVID-19) outbreak in China: summary of a report of 72314 cases from the Chinese Center for Disease Control and Prevention. JAMA 323, 1239-1242 (2020).

71. Dudley, J. P. \& Lee, N. T. Disparities in age-specific morbidity and mortality from SARS-CoV-2 in China and the Republic of Korea. Clin. Infect. Dis. 71, 863-865 (2020).

72. Conti, P. \& Younes, A. Coronavirus COV-19/SARS-CoV-2 affects women less than men: clinical response to viral infection. J. Biol. Regul. Homeost. Agents 34, 339-343 (2020).

73. Shah, M., Sachdeva, M. \& Dodiuk-Gad, R. P. COVID-19 and racial disparities. J. Am. Acad. Dermatol. 83, e35 (2020).

74. McCoy, J. et al. Racial variations in COVID-19 deaths may be due to androgen receptor genetic variants associated with prostate cancer and androgenetic alopecia. Are anti-androgens a potential treatment for COVID-19? J. Cosmet. Dermatol. 19, 1542-1543 (2020).

75. Laurencin, C. T. \& McClinton, A. The COVID-19 pandemic: a call to action to identify and address racial and ethnic disparities. J. Racial Ethn. Health Disparities https://doi.org/10.1007/s40615-020-00756-0 (2020).

76. Brake, S. J. et al. Smoking upregulates angiotensin-converting enzyme-2 receptor: a potential adhesion site for novel coronavirus SARS-CoV-2 (Covid-19). J. Clin. Med. 9, https://doi.org/10.3390/jcm9030841 (2020).

77. Cai, G., Bosse, Y., Xiao, F., Kheradmand, F. \& Amos, C. I. Tobacco smoking increases the lung gene expression of ACE2, the receptor of SARS-CoV-2. Am. J. Respir. Crit. Care Med. 201, 1557-1559 (2020).

78. Lukassen, $\mathrm{S}$. et al. SARS-CoV-2 receptor ACE2 and TMPRSS2 are primarily expressed in bronchial transient secretory cells. EMBO J. 39, e105114 (2020).

79. Afzal, A. Molecular diagnostic technologies for COVID-19: limitations and challenges. J. Adv. Res. 26, 149-159 (2020).

80. Kevadiya, B. D. et al. Diagnostics for SARS-CoV-2 infections. Nat. Mater. 20, 593-605 (2021).

81. Liu, W. et al. Evaluation of nucleocapsid and spike protein-based enzyme-linked immunosorbent assays for detecting antibodies against SARS-CoV-2. J. Clin. Microbiol. 58, https://doi.org/10.1128/JCM.00461-20 (2020)

82. Zhao, J. et al. Antibody responses to SARS-CoV-2 in patients with novel coronavirus disease 2019. Clin. Infect. Dis. 71, 2027-2034 (2020).

83. Li, Z. et al. Development and clinical application of a rapid IgM-IgG combined antibody test for SARS-CoV-2 infection diagnosis. J. Med Virol. 92, 1518-1524 (2020).

84. Zhao, G. et al. A safe and convenient pseudovirus-based inhibition assay to detect neutralizing antibodies and screen for viral entry inhibitors against the novel human coronavirus MERS-CoV. Virol. J. 10, 266 (2013).

85. Li, Q., Liu, Q., Huang, W., Li, X. \& Wang, Y. Current status on the development of pseudoviruses for enveloped viruses. Rev. Med. Virol. 28, https://doi.org/10.1002/rmv.1963 (2018).

86. Letko, M., Marzi, A. \& Munster, V. Functional assessment of cell entry and receptor usage for SARS-CoV-2 and other lineage B betacoronaviruses. Nat. Microbiol 5, 562-569 (2020).

87. Matsuyama, S. et al. Enhanced isolation of SARS-CoV-2 by TMPRSS2expressing cells. Proc. Natl Acad. Sci. USA 117, 7001-7003 (2020).

88. Cao, X. et al. Invited review: human air-liquid-interface organotypic airway tissue models derived from primary tracheobronchial epithelial cells-overview and perspectives. Vitr. Cell Dev. Biol. Anim. 57, 104-132 (2021).

89. Schoch, K. G. et al. A subset of mouse tracheal epithelial basal cells generates large colonies in vitro. Am. J. Physiol. Lung Cell. Mol. Physiol. 286, L631-L642 (2004).

90. Rock, J. R. et al. Basal cells as stem cells of the mouse trachea and human airway epithelium. Proc. Natl Acad. Sci. USA 106, 12771-12775 (2009).

91. Ettayebi, K. et al. Replication of human noroviruses in stem cell-derived human enteroids. Science 353, 1387-1393 (2016).

92. Sasai, Y. Next-generation regenerative medicine: organogenesis from stem cells in 3D culture. Cell Stem Cell 12, 520-530 (2013). 
93. Wong, A. P. et al. Directed differentiation of human pluripotent stem cells into mature airway epithelia expressing functional CFTR protein. Nat. Biotechnol. 30, 876-882 (2012).

94. Sachs, N. et al. Long-term expanding human airway organoids for disease modeling. EMBO J. 38, https://doi.org/10.15252/embj.2018100300 (2019).

95. Chen, K. G., Zhong, P., Zheng, W. \& Beekman, J. M. Pharmacological analysis of CFTR variants of cystic fibrosis using stem cell-derived organoids. Drug Disco. Today 24, 2126-2138 (2019).

96. Basil, M. C. et al. The cellular and physiological basis for lung repair and regeneration: past, present, and future. Cell Stem Cell 26, 482-502 (2020).

97. Hogan, B. L. et al. Repair and regeneration of the respiratory system: complexity, plasticity, and mechanisms of lung stem cell function. Cell Stem Cell 15, 123-138 (2014).

98. Nadkarni, R. R., Abed, S. \& Draper, J. S. Organoids as a model system for studying human lung development and disease. Biochem. Biophys. Res. Commun. 473, 675-682 (2016)

99. Gkatzis, K., Taghizadeh, S., Huh, D., Stainier, D. Y. R. \& Bellusci, S. Use of three-dimensional organoids and lung-on-a-chip methods to study lung development, regeneration and disease. Eur. Respir. J. 52, https://doi. org/10.1183/13993003.00876-2018 (2018).

100. Huang, S. X. et al. Efficient generation of lung and airway epithelial cells from human pluripotent stem cells. Nat. Biotechnol. 32, 84-91 (2014).

101. Chen, Y. W. et al. A three-dimensional model of human lung development and disease from pluripotent stem cells. Nat. Cell Biol. 19, 542-549 (2017).

102. Porotto, M. et al. Authentic modeling of human respiratory virus infection in human pluripotent stem cell-derived lung organoids. mBio 10, https:// doi.org/10.1128/mBio.00723-19 (2019).

103. Yamamoto, Y. et al. Long-term expansion of alveolar stem cells derived from human iPS cells in organoids. Nat. Methods 14, 1097-1106 (2017).

104. Strikoudis, A. et al. Modeling of fibrotic lung disease using 3D organoids derived from human pluripotent stem cells. Cell Rep. 27, 3709-3723 (2019).

105. Dye, B. R. et al. In vitro generation of human pluripotent stem cell derived lung organoids. eLife 4, e05098 (2015).

106. McCauley, K. B. et al. Efficient derivation of functional human airway epithelium from pluripotent stem cells via temporal regulation of Wnt signaling. Cell Stem Cell 20, 844-857 e846 (2017).

107. Miller, A. J. et al. Generation of lung organoids from human pluripotent stem cells in vitro. Nat. Protoc. 14, 518-540 (2019).

108. Barkauskas, C. E. et al. Lung organoids: current uses and future promise. Development 144, 986-997 (2017).

109. Sungnak, W. et al. SARS-CoV-2 entry factors are highly expressed in nasal epithelial cells together with innate immune genes. Nat. Med. 26, 681-687 (2020).

110. Barkauskas, C. E. et al. Type 2 alveolar cells are stem cells in adult lung. J. Clin. Invest. 123, 3025-3036 (2013)

111. Ziegler, C. G. K. et al. SARS-CoV-2 receptor ACE2 is an interferon-stimulated gene in human airway epithelial cells and is detected in specific cell subsets across tissues. Cell 181, 1016-1035 (2020).

112. Youk, J. et al. Three-dimensional human alveolar stem cell culture models reveal infection response to SARS-CoV-2. Cell Stem Cell 27, 905-919 (2020).

113. Schaefer, I. M. et al. In situ detection of SARS-CoV-2 in lungs and airways of patients with COVID-19. Mod. Pathol. 33, 2104-2114 (2020).

114. Borczuk, A. C. et al. COVID-19 pulmonary pathology: a multi-institutional autopsy cohort from Italy and New York City. Mod. Pathol. 33, 2156-2168 (2020).

115. Mirabelli, C. et al. Morphological cell profiling of SARS-CoV-2 infection identifies drug repurposing candidates for COVID-19. Preprint at bioRxiv https://doi.org/10.1101/2020.05.27.117184 (2020).

116. Abo, K. M. et al. Human iPSC-derived alveolar and airway epithelial cells can be cultured at air-liquid interface and express SARS-CoV-2 host factors. Preprint at bioRxiv https://doi.org/10.1101/2020.06.03.132639 (2020).

117. Huang, J. et al. SARS-CoV-2 infection of pluripotent stem cell-derived human lung alveolar type 2 cells elicits a rapid epithelial-intrinsic inflammatory response. Cell Stem Cell 27, 962-973 (2020).

118. Duan, F. et al. Modeling COVID-19 with human pluripotent stem cell-derived cells reveals synergistic effects of anti-inflammatory macrophages with ACE2 inhibition against SARS-CoV-2. Res. Sq. https:// doi.org/10.21203/rs.3.rs-62758/v1 (2020).

119. Lee, A. S., Tang, C., Rao, M. S., Weissman, I. L. \& Wu, J. C. Tumorigenicity as a clinical hurdle for pluripotent stem cell therapies. Nat. Med. 19, 998-1004 (2013)

120. Gore, A. et al. Somatic coding mutations in human induced pluripotent stem cells. Nature 471, 63-67 (2011).

121. Merkle, F. T. et al. Human pluripotent stem cells recurrently acquire and expand dominant negative P53 mutations. Nature 545, 229-233 (2017).

122. Baker, D. E. et al. Adaptation to culture of human embryonic stem cells and oncogenesis in vivo. Nat. Biotechnol. 25, 207-215 (2007).
123. Mayshar, Y. et al. Identification and classification of chromosomal aberrations in human induced pluripotent stem cells. Cell Stem Cell 7, 521-531 (2010).

124. Rodrigues Toste de Carvalho, A. L. et al. The in vitro multilineage differentiation and maturation of lung and airway cells from human pluripotent stem cell-derived lung progenitors in 3D. Nat. Protoc. 16, 1802-1829 (2021)

125. Studer, L., Vera, E. \& Cornacchia, D. Programming and reprogramming cellular age in the era of induced pluripotency. Cell Stem Cell 16, 591-600 (2015).

126. Hawkins, F. J. et al. Derivation of airway basal stem cells from human pluripotent stem cells. Cell Stem Cell 28, 79-95 (2021).

127. Mulay, A. et al. SARS-CoV-2 infection of primary human lung epithelium for COVID-19 modeling and drug discovery. Cell Rep. 35, 109055 (2021).

128. Takebe, T. \& Wells, J. M. Organoids by design. Science 364, 956-959 (2019).

129. Dye, B. R. et al. A bioengineered niche promotes in vivo engraftment and maturation of pluripotent stem cell derived human lung organoids. eLife $\mathbf{5}$, e19732 (2016)

130. van Velthoven, C. T. J. \& Rando, T. A. Stem cell quiescence: dynamism, restraint, and cellular idling. Cell Stem Cell 24, 213-225 (2019).

131. Graf, T. \& Stadtfeld, M. Heterogeneity of embryonic and adult stem cells. Cell Stem Cell 3, 480-483 (2008).

132. Wagers, A. J. \& Weissman, I. L. Plasticity of adult stem cells. Cell 116, 639-648 (2004).

133. Nabhan, A. N., Brownfield, D. G., Harbury, P. B., Krasnow, M. A. \& Desai, T. J. Single-cell Wnt signaling niches maintain stemness of alveolar type 2 cells. Science 359, 1118-1123 (2018).

134. Choi, J. et al. Inflammatory signals induce AT2 cell-derived damage-associated transient progenitors that mediate alveolar regeneration. Cell Stem Cell 27, 366-382.e7 (2020).

135. Zacharias, W. J. et al. Regeneration of the lung alveolus by an evolutionarily conserved epithelial progenitor. Nature 555, 251-255 (2018).

136. Salahudeen, A. A. et al. Progenitor identification and SARS-CoV-2 infection in human distal lung organoids. Nature 588, 670-675 (2020).

137. Tindle, C. et al. Adult stem cell-derived complete lung organoid models emulate lung disease in COVID-19. Preprint at bioRxiv https://doi. org/10.1101/2020.10.17.344002 (2020).

138. van der Vaart, J. \& Clevers, H. Airway organoids as models of human disease. J. Intern Med 289, 604-613 (2021).

139. Evangelou, K. et al. SARS-CoV-2 infects lung epithelial cells and induces senescence and an inflammatory response in patients with severe COVID-19. Preprint at bioRxiv https://doi.org/10.1101/2021.01.02.424917 (2021).

140. Hillel-Karniel, C. et al. Multi-lineage lung regeneration by stem cell transplantation across major genetic barriers. Cell Rep. 30, 807-819.e4 (2020).

141. Weiner, A. I. et al. Mesenchyme-free expansion and transplantation of adult alveolar progenitor cells: steps toward cell-based regenerative therapies. NPJ Regen. Med 4, 17 (2019).

142. Dorrello, N. V. et al. Functional vascularized lung grafts for lung bioengineering. Sci. Adv. 3, e1700521 (2017)

143. Boehm, M. \& Nabel, E. G. Angiotensin-converting enzyme 2-a new cardiac regulator. N. Engl. J. Med. 347, 1795-1797 (2002).

144. Zhang, H., Penninger, J. M., Li, Y., Zhong, N. \& Slutsky, A. S. Angiotensin-converting enzyme 2 (ACE2) as a SARS-CoV-2 receptor: molecular mechanisms and potential therapeutic target. Intensive Care Med 46, 586-590 (2020).

145. Abassi, Z., Assady, S., Khoury, E. E. \& Heyman, S. N. Letter to the Editor: Angiotensin-converting enzyme 2: an ally or a Trojan horse? Implications to SARS-CoV-2-related cardiovascular complications. Am. J. Physiol. Heart Circ. Physiol. 318, H1080-H1083 (2020).

146. Ferrario, C. M. et al. Effect of angiotensin-converting enzyme inhibition and angiotensin II receptor blockers on cardiac angiotensin-converting enzyme 2. Circulation 111, 2605-2610 (2005).

147. Jacob, A. et al. Differentiation of human pluripotent stem cells into functional lung alveolar epithelial cells. Cell Stem Cell 21, 472-488.e10 (2017).

148. Korogi, Y. et al. In vitro disease modeling of Hermansky-Pudlak syndrome type 2 using human induced pluripotent stem cell-derived alveolar organoids. Stem Cell Rep. 12, 431-440 (2019).

149. Tan, Q., Choi, K. M., Sicard, D. \& Tschumperlin, D. J. Human airway organoid engineering as a step toward lung regeneration and disease modeling. Biomaterials 113, 118-132 (2017).

150. Lee, J. H. et al. Lung stem cell differentiation in mice directed by endothelial cells via a BMP4-NFATc1-thrombospondin-1 axis. Cell $\mathbf{1 5 6}$ 440-455 (2014).

151. Niethamer, T. K. et al. Defining the role of pulmonary endothelial cell heterogeneity in the response to acute lung injury. eLife $\mathbf{9}$, e53072 (2020) 
152. Ding, B. S. et al. Endothelial-derived angiocrine signals induce and sustain regenerative lung alveolarization. Cell 147, 539-553 (2011).

153. Rafii, S. et al. Platelet-derived SDF-1 primes the pulmonary capillary vascular niche to drive lung alveolar regeneration. Nat. Cell Biol. 17, 123-136 (2015).

154. Tanaka, T., Narazaki, M. \& Kishimoto, T. Immunotherapeutic implications of IL-6 blockade for cytokine storm. Immunotherapy 8, 959-970 (2016).

155. Takebe, T. et al. Vascularized and functional human liver from an iPSC-derived organ bud transplant. Nature 499, 481-484 (2013).

156. Cakir, B. et al. Engineering of human brain organoids with a functional vascular-like system. Nat. Methods 16, 1169-1175 (2019).

157. Lin, L., Lu, L., Cao, W. \& Li, T. Hypothesis for potential pathogenesis of SARS-CoV-2 infection-a review of immune changes in patients with viral pneumonia. Emerg. Microbes Infect. 9, 727-732 (2020).

158. Fadini, G. P., Morieri, M. L., Longato, E. \& Avogaro, A. Prevalence and impact of diabetes among people infected with SARS-CoV-2. J. Endocrinol. Invest 43, 867-869 (2020).

159. Krammer, F. SARS-CoV-2 vaccines in development. Nature $\mathbf{5 8 6}$ 516-527 (2020).

160. Purwada, A. \& Singh, A.Immuno-engineered organoids for regulating the kinetics of B-cell development and antibody production. Nat. Protoc. 12, 168-182 (2017).

161. Hoffmann, M., Kleine-Weber, H. \& Pohlmann, S. A multibasic cleavage site in the spike protein of SARS-CoV-2 is essential for infection of human lung cells. Mol. Cell 78, 779-784.e5 (2020).

162. Shang, J. et al. Cell entry mechanisms of SARS-CoV-2. Proc. Natl Acad. Sci. USA 117, 11727-11734 (2020).
163. Zepp, J. A. et al. Distinct mesenchymal lineages and niches promote epithelial self-renewal and myofibrogenesis in the lung. Cell 170, 1134-1148.e10 (2017).

164. Lee, J. H. et al. Anatomically and functionally distinct lung mesenchymal populations marked by Lgr5 and Lgr6. Cell 170, 1149-1163.e12 (2017).

\section{Acknowledgements}

This work was supported by the Intramural Research Program of the National Institutes of Health at the National Institute of Neurological Disorders and Stroke (K.G.C. and K.P.). J.R.S. was supported by the National Heart, Lung, and Blood Institute of the National Institutes of Health (grant number R01HL119215).

\section{Competing interests}

The authors declare no competing interests.

\section{Additional information}

Correspondence should be addressed to K.G.C.

Peer review information Nature Cell Biology thanks the anonymous reviewers for their contribution to the peer review of this work.

Reprints and permissions information is available at www.nature.com/reprints.

Publisher's note Springer Nature remains neutral with regard to jurisdictional claims in published maps and institutional affiliations.

This is a U.S. government work and not under copyright protection in the U.S.; foreign copyright protection may apply 2021 